\title{
A new perspective of Ferroptosis in People with Psoriasis and Psoriatic Arthritis basing on a bioinformatics analysis
}

\section{Xiaobin Li}

The Second Affiliated Hospital of Zhejiang University School of Medicine

Xiaohua Tao

Zhejiang Provincial People's Hospital, Hangzhou Medical College

Xiaoxia Ding ( $\sim$ dingxx93@163.com )

Zhejiang Provincial People's Hospital, Hangzhou Medical College

\section{Research Article}

Keywords: psoriasis, psoriasis arthritis, ferroptosis, cancer risk, bioinformatics

Posted Date: February 24th, 2022

DOI: https://doi.org/10.21203/rs.3.rs-1333902/v1

License: (c) (i) This work is licensed under a Creative Commons Attribution 4.0 International License.

Read Full License 


\section{Abstract}

Patients with psoriasis (Ps) and psoriatic arthritis (PsA) are reported with increased cancer risk, but the underlying correlation in less clear. This study was performed to identify the possible association of ferroptosis with Ps and PsA using bioinformatics methods. Differentially expressed genes (DEGs) were identified between blood samples from patients with PsA and Ps as well as normal control (CL), and the overlapped as well as merged DEGS between the two sets were used for further analysis including the hub genes exploration, enrichment analysis, interaction network construction and engaged pathway analysis. The expression level of ferroptosis related genes among above two sets were performed and the association of key ferroptosis related genes and hub genes was explored to clarify whether ferroptosis correlated with Ps and PsA. The findings revealed a correlation between ferroptosis regulatory genes and hub genes in both Ps and PsA groups, and it appeared to be stronger in the PsA group. Furthermore, most of the correlations are negative. And the similar results were found in the validation dataset. In conclusion, ferroptosis may be a potential research point in psoriasis and psoriasis arthritis development and increased cancer risk.

\section{Introduction}

Psoriasis (Ps) is a hyperproliferative chronic inflammatory skin and joints disease with unknown etiology ${ }^{1}$, which affects $2-3 \%$ of the population. Psoriatic arthritis (PsA), occurring in up to $30 \%$ of people with psoriasis, is considered to be more severely affected ${ }^{2}$. A number of comorbid conditions, such as depression, cardiovascular disease, and particularly cancer, compound the impact of Ps and PsA. The incidence of cancer and cancer-related mortality seem to be higher in people with Ps and PsA, but the underlying association is much less clear ${ }^{3,4}$. To improve the understanding of the underlying mechanisms of this increased risk, further research is needed.

Ferroptosis, a new form of regulated cell death that is iron- and reactive oxygen species (ROS)-dependent, has attracted much attention in cancer development and treatment ${ }^{5}$. According to research, ferroptosis is likely to inhibit tumor formation and/or progression, which means inducing ferroptosis is a promising anticancer strategy. Activation of lipid ROS and iron metabolism or suppression of antioxidant metabolism is the primary approach of ferroptosis induction based cancer therapy ${ }^{6,7}$. Interestingly, ROS occurs systemically and locally in psoriasis ${ }^{8}$, many current treatments further increase oxidative stress and ROS production to accelerate the removal of damaged epidermal cells ${ }^{9}$. So far, no studies have investigated the association between Ps development and ferroptosis, which may provide a new perspective on the cancer prevention and treatment in patients with psoriasis and psoriasis arthritis.

There is growing interest in microarray platforms as a way to detect genetic alterations and to determine biomarkers for many diseases ${ }^{10}$. Several biomarkers and pathways have been implicated in the development of psoriasis in previous studies on microarray data ${ }^{11,12}$, but still no studies focus on the link between ferroptosis and psoriasis. In this study, we compared the gene expression of patients with 
psoriasis arthritis or psoriasis and normal person by integrated bioinformatics methods. The DEGs overlapping and merging from the two comparisons were used for further analysis. More importantly, we analyzed the expression level of ferroptosis regulators in Ps and PsA group. Consequently, the association of key ferroptosis regulators and hub genes was explored. The specific process can be seen in Fig. 1. We found the correlation between hub genes and key ferroptosis regulators were negative. Additionally, another dataset from GEO was chosen for validation and similar results were obtained, which further supported the hypothesis that ferroptosis may correlated with the disease development and high cancer risk in patients with psoriasis.

\section{Material And Methods Dataset Collection}

Gene expression datasets were collected from the Gene Expression Omnibus database (http://www.ncbi.nlm.nih.gov/geo) $)^{13}$.

\section{Data processing and Differential Expression Analysis}

Raw data were processed and analyzed using R (version 4.0.2). Firstly, the the RMA algorithm was used for background correction and data normalization and the result was show through box plot. Then, the "limma R" package was used to identify DEGs ${ }^{14}(|\log 2 \mathrm{FC}|>1, \mathrm{p}<0.05)$ between each group in the dataset, and a volcano map and heatmap of DEGs was drawn respectively.

\section{Functional Correlation Analysis}

Gene Ontology (GO) and Gene Set Enrichment Analysis (GSEA) on DEGs were performed respectively using "clusterProfiler" package ${ }^{15}$ "c2.cp.kegg.v7.0.symbols.gmt" was selected as the reference gene set. Kyoto Encyclopedia of Genes and Genomes (KEGG) pathway analyses were performed using GAGE ${ }^{16}$ followed by visualization with the ggplot2 $\mathrm{R}$ package. Annotation of the genes and pathways was provided by the KEGG database (http://www.genome.jp/kegg). Disease Ontology (DO) enrichment analysis was performed with an online tool Enrichr (https://maayanlab.cloud/Enrichr/). A false discovery rate $(F D R)<0.25$ and $p<0.05$ were considered significant enrichment.

\section{Construction of the PPI network}

To characterize the crucial DEGs and clusters in skin scar, we used an online tool STRING (https://string$\mathrm{db}$. org/) to construct PPI networks with a minimum required interaction score of $4^{17}$. For further analysis, Cytoscape software was used with the download interaction information. Significant genes were determined by the cytoHubba plugin as hub genes ${ }^{18}$.

\section{Analysis of related Ferroptosis regulators in keloid and Ferroptosis -Related genes among hub genes}


The list of genes involved in ferroptosis was retrieved from a review on this topic ${ }^{19}$. Then, differential expression analysis between patients with psoriatic arthritis, psoriasis and normal person were conducted respectively on selected genes and key ferroptosis regulators were obtained. Finally, associations between those key ferroptosis regulators and top 10 hub genes were explored.

\section{Validation of association between related Ferroptosis regulators and hub genes}

To validate the association between those key ferroptosis regulators and hub genes, we performed the same association analysis in 44 blood samples of psoriasis from GSE145725 dataset.

\section{Results}

\section{Data preprocessing}

After searching in Gene Expression Omnibus database with the inclusion criteria included: (1) patients with psoriatic arthritis or psoriasis; (2) whole blood, 2 datasets (GSE61281 and GSE55201) were obtained. For details, 52 blood samples from GSE61281 (20 from patients with psoriatic arthritis,20 from psoriasis and 12 normal person); 44 blood samples of psoriasis from GSE145725 were used only for validation.

After obtaining the datasets, we firstly performed background correction and data normalization, and the result is presented in box plots (Fig. 2).

\section{Identification of DEGS}

After data preprocessing, DEGs were identified with the setting of cutoff at FDR $<0.05$ and $\mid \log 2$ (FC) $\mid>1$. As shown in the volcano plot, totally 86 DEGs in the comparison of PsA and Ps, and 273 DEGs in the comparison of PsA and normal person were obtained (Fig. 3A, B). The top 50 DEGs of two sets are illustrated in two heatmap plots (Fig. 3C, D). The 28 overlapped DEGs between the two sets are presented in a Venn diagram (Fig. 3E).

\section{Functional Correlation Analysis}

Enriched GO terms were divided into three categories: BP, CC, and MF. As shown in Fig. 4C, the DEGs merged from the comparison of PsA, Ps and CL were mainly enriched in the 'protein targeting to ER', 'cotranslational protein targeting to membrane' and 'SRP-dependent cotranslational protein targeting to membrane' in the BP group. CC analysis indicated that the DEGs were mainly enriched in 'cytosolic large ribosomal subunit', 'ribosomal subunit' and 'cytosolic ribosome'. In terms of MF, DEGs were most enriched in 'mRNA 5'-UTR binding' and 'structural constituent of ribosome'. KEGG analysis of DEGs revealed that they were mainly enriched in 'Ribosome'. Other results of DEGS from different comparison were shown in Fig. $4 \mathrm{~A}$ and $\mathrm{B}$. 
GSEA and DO results are shown in Fig. 5. In the set of PsA and Ps, the enriched pathways mainly involved 'signaling by receptor tyrosine kinases' and 'VEGFavegfr2 signaling pathway' (Fig. 5B). While in the set of PsA and CL, 'metabolism of amino acids and derivatives' and 'translation' were mainly involved (Fig. 5A). The DO analysis results are shown in Fig. 5C. Diseases enriched by overlapped DEGs mainly included Osteoarthritis of hip and Renal cancer metastatic.

\section{PPI Network Construction}

The PPI network was constructed using STRING to investigate the interaction of overlapped DEGs, and then the result was visualized in Cytoscape. Subsequently, cytoHubba plugin was used to characterize the hub genes. The top 10 genes based on the filtering algorithm (degree) were shown in Fig. 6.

\section{Expression Profile of Selected Ferroptosis Regulators}

Differences in the expression levels of 24 selected ferroptosis regulators were compared between PsA, Ps and CL. Compared with expression in Ps, ACSL4, CISD1, EMC2 and SAT1 expression levels were increased, while CARS was decreased expressed in PsA group (Fig. 7B). While compared with CL, the expression level of CISD1, CS, EMC2 were significantly increased (Fig. 7A), but CARS, FDFT1 and HSPA5 were decreased. These results revealed that ferroptosis may correlated with psoriasis and psoriasis arthritis development, and the underlying mechanism needs to be further explored in the future.

\section{Association between key ferroptosis regulators and hub genes}

We then tried to investigate the correlation between top 10 hub genes and the key ferroptosis regulators. Limited by the platform raw data, totally 3 ferroptosis regulators and 7 hub genes participated this analysis. As shown in Fig.7 C, in PsA group, among the 3 key ferroptosis regulators, SAT1 showed negative association with all 7 hub genes, ACSL4 also showed a negative association with all 7 hub genes, but only 4 significantly. CARS showed positive association with 2 hub genes (CLEC12B AND LPAR6). And the association analysis result in Ps group was shown in Fig.7D.

\section{Association analysis validation}

As shown in Fig. 8, similar negative associations between 3 ferroptosis regulators and 7 hub genes were found, which further clarify the potential role of ferroptosis in the increased risk of cancer in patients with psoriasis and psoriasis arthritis.

\section{Discussion}

The risk of cancer developing in people with psoriasis has raised some concern ${ }^{20,21}$. The risk of cancer incidence is also debated with patients inflicted by psoriatic arthritis, who are considered to be more severely affected than those suffering from psoriasis ${ }^{22}$. Contrary to the established nature of the associations between these comorbidities including depression and cardiovascular disease, cancer and psoriasis appear to be much less well-established ${ }^{23}$. 
Iron is an important factor in cancer development. In various types of cancers, proteins that are essential to biological events require iron for their function ${ }^{24}$. Ferroptosis is a novel iron-dependent programmed cell death process, which is characteristically distinct from autophagy, apoptosis, and other forms of necrosis ${ }^{25}$. The importance of ferroptosis in disease has been reported a lot, especially in cancer ${ }^{26}$. Research into the association of ferroptosis with psoriasis and psoriatic arthritis may provide new insights into the high risk of cancer in patients with Ps and PsA.

In this study, we found a correlation between ferroptosis regulatory genes and hub genes in both psoriasis and psoriatic arthritis groups, and it appeared to be stronger in the PsA group. Furthermore, most of the correlations are negative. And the similar results were found in the validation dataset. We therefore propose the conjecture that in patients with psoriasis and psoriatic arthritis, the level of ferroptosis may be lower than normal subjects, which further contributing to the high risk of cancer. Deeper research is still needed to confirm the potential mechanism and exact association.

\section{Declarations}

\section{Author Contributions}

Xiaohua Tao and Xiaobin Li: study concept and design. Xiaoxia Ding and Xiaobin Li: data analysis and manuscript drafting. All authors reviewed and approved the manuscript prior to its submission.

\section{Conflict of Interest}

The authors declare that the research was conducted in the absence of any commercial or financial relationships that could be construed as a potential conflict of interest.

\section{Availability of Data and Materials}

The datasets generated and analysed during the current study are available in the Gene Expression Omnibus database『http://www.ncbi.nlm.nih.gov/geo》

\section{References}

1. Karrys, A. et al. Bioactive Dietary VDR Ligands Regulate Genes Encoding Biomarkers of Skin Repair That Are Associated with Risk for Psoriasis. Nutrients 10, 174 (2018).

2. Coates, L. C. et al. Effect of tight control of inflammation in early psoriatic arthritis (TICOPA): a UK multicentre, open-label, randomised, controlled trial. Lancet 386, 2489-2498 (2015).

3. Trafford, A. M., Parisi, R., Kontopantelis, E., Griffiths, C. E. M. \& Ashcroft, D. M. Association of Psoriasis With the Risk of Developing or Dying of Cancer. JAMA Dermatol 155, 1390-1403 (2019).

4. Vaengebjerg, S., Skov, L., Egeberg, A. \& Loft, N. D. Prevalence, Incidence, and Risk of Cancer in Patients With Psoriasis and Psoriatic Arthritis. JAMA Dermatol 156, 421-429 (2020). 
5. Shen, Z. et al. Emerging Strategies of Cancer Therapy Based on Ferroptosis. Adv Mater 30, e1704007 (2018).

6. Gao, M. et al. Ferroptosis is an autophagic cell death process. Cell Res 26, 1021-1032 (2016).

7. Luo, Y. et al. Regulation of ferroptosis by non-coding RNAs in the development and treatment of cancer. Oncol Rep 45, 29-48 (2021).

8. Ascenso, A., Ribeiro, H. M., Marques, H. C. \& Simoes, S. Topical delivery of antioxidants. Curr Drug Deliv 8, 640-660 (2011).

9. Jarocka-Karpowicz, I., Biernacki, M., Wroński, A., Gęgotek, A. \& Skrzydlewska, E. Cannabidiol Effects on Phospholipid Metabolism in Keratinocytes from Patients with Psoriasis Vulgaris. Biomolecules 10,367 (2020).

10. Su, Z. et al. An investigation of biomarkers derived from legacy microarray data for their utility in the RNA-seq era. Genome Biol 15, 3273 (2014).

11. Li, H. et al. Identification of potential key mRNAs and LncRNAs for psoriasis by bioinformatic analysis using weighted gene co-expression network analysis. Mol Genet Genomics 295, 741-749 (2020).

12. Naderi, R., Saadati Mollaei, H., Elofsson, A. \& Hosseini Ashtiani, S. Using Micro- and Macro-Level Network Metrics Unveils Top Communicative Gene Modules in Psoriasis. Genes (Basel) 11, 914 (2020).

13. Clough, E. \& Barrett, T. The Gene Expression Omnibus database. Methods Mol Biol 1418, 93-110 (2016).

14. Ritchie, M. E. et al. limma powers differential expression analyses for RNA-sequencing and microarray studies. Nucleic Acids Res 43, e47 (2015).

15. Yu, G., Wang, L.-G., Han, Y. \& He, Q.-Y. clusterProfiler: an R package for comparing biological themes among gene clusters. OMICS 16, 284-287 (2012).

16. Luo, W., Friedman, M. S., Shedden, K., Hankenson, K. D. \& Woolf, P. J. GAGE: generally applicable gene set enrichment for pathway analysis. BMC Bioinformatics 10, 161 (2009).

17. Szklarczyk, D. et al. STRING v11: protein-protein association networks with increased coverage, supporting functional discovery in genome-wide experimental datasets. Nucleic Acids Res 47, D607D613 (2019).

18. Chin, C.-H. et al. cytoHubba: identifying hub objects and sub-networks from complex interactome. BMC Syst Biol 8, S11 (2014).

19. Zhou, N. \& Bao, J. FerrDb: a manually curated resource for regulators and markers of ferroptosis and ferroptosis-disease associations. Database (Oxford) 2020, baaa021 (2020).

20. Alinaghi, F. et al. Prevalence of psoriatic arthritis in patients with psoriasis: A systematic review and meta-analysis of observational and clinical studies. J Am Acad Dermatol 80, 251-265.e19 (2019).

21. Šahmatova, L. et al. Signs of innate immune activation and premature immunosenescence in psoriasis patients. Sci Rep 7, 7553 (2017). 
22. Hagberg, K. W. et al. Rates of Cancers and Opportunistic Infections in Patients With Psoriatic Arthritis Compared With Patients Without Psoriatic Arthritis. J Clin Rheumatol 22, 241-247 (2016).

23. Guenther, L. \& Gulliver, W. Psoriasis comorbidities. J Cutan Med Surg 13 Suppl 2, S77-87 (2009).

24. Raza, M., Chakraborty, S., Choudhury, M., Ghosh, P. C. \& Nag, A. Cellular iron homeostasis and therapeutic implications of iron chelators in cancer. Curr Pharm Biotechnol 15, 1125-1140 (2014).

25. Lei, G. et al. The role of ferroptosis in ionizing radiation-induced cell death and tumor suppression. Cell Res 30, 146-162 (2020).

26. Stockwell, B. R. et al. Ferroptosis: a regulated cell death nexus linking metabolism, redox biology, and disease. Cell 171, 273-285 (2017).

\section{Figures}




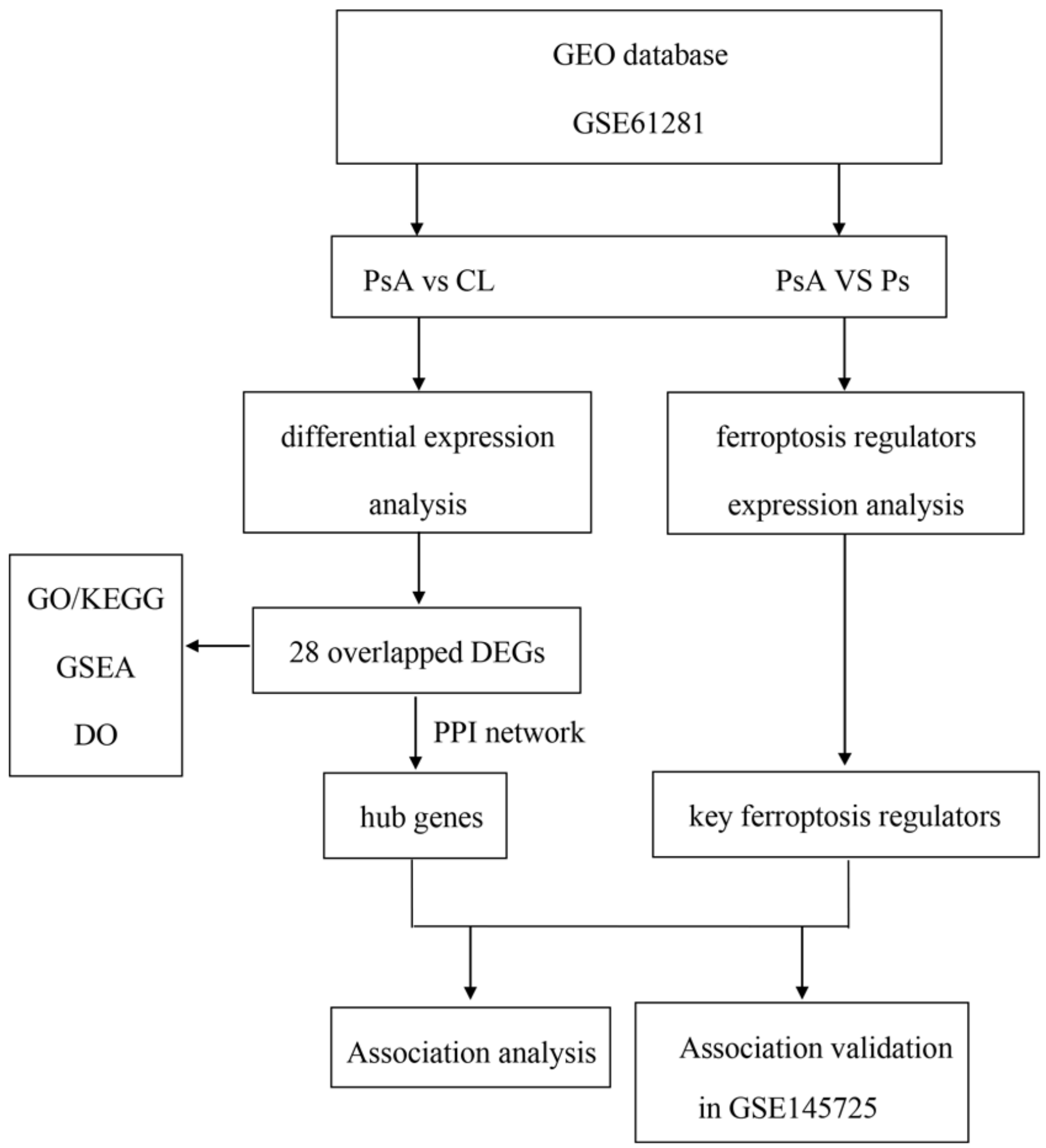

Figure 1

Flowchart of Methods 
A

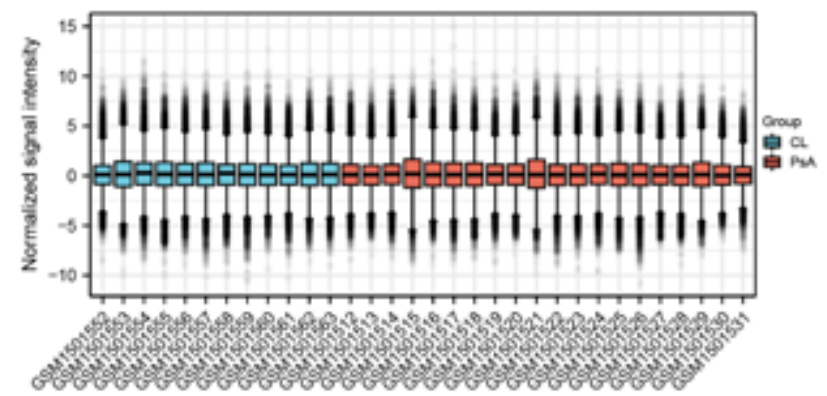

B

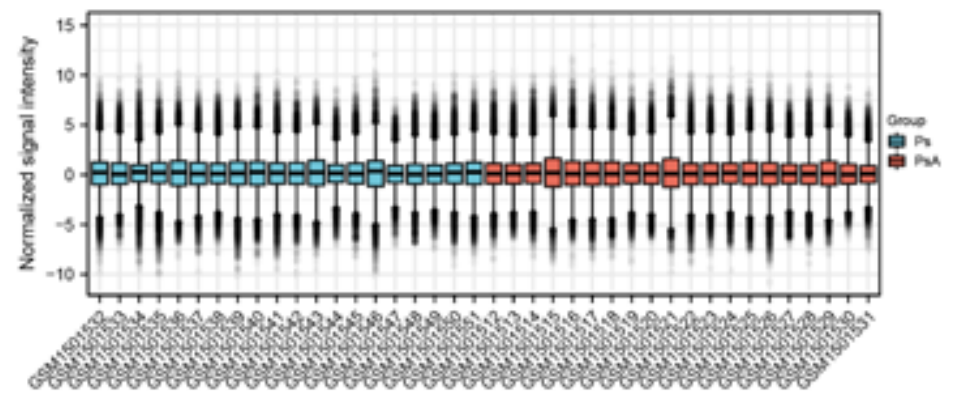

Figure 2

Normalization of microarray dataset. A: Box plot after data standardization of PsA and CL group. B: Box plot after data standardization of PsA and Ps group.

\section{Figure 3}

Identification of DEGS. Volcano plots of DEGS between the PSA and the CL (A) as well as PsA and Ps group (B). The red point in the plot represents the over-expressed mRNAs and the blue point indicates the down-expressed mRNAs with statistical significance. Heatmap of top 50 DEGs between PsA and CL group (C) and between the PsA and Ps group (D). E: A Venn plot of DEGs overlapped between two groups.

\section{Figure 4}

KEGG enrichment analysis and Gene ontology (GO) analysis of DEGs. A: Enriched KEGG pathways and GO analysis in PsA and CL group. B: Enriched KEGG pathways and GO analysis in PsA and Ps group. C: Enriched KEGG pathways and GO analysis of merged DEGS.

In the enrichment result, $p<0.05$ or FDR $<0.05$ is considered to be enriched to a meaningful pathway. BP: The biological process; CC: cellular component; MF: molecular function.

\section{Figure 5}

Gene set enrichment and Disease Ontology (DO) analysis. A: GSEA results of PsA and CL group; B: GSEA results of PsA and Ps group; C: Disease Ontology (DO) analysis of overlapped DEGS. 


\section{Figure 6}

PPI network and interaction analysis. A: PPI network of overlapped DEGs. Red and blue circles represented up- and downregulated genes, respectively. The edge color and width are proportional to the combined score analyzed by STRING. B: The top 10 hub genes determined by cytoHubba.

\section{Figure 7}

Ferroptosis related analysis. The expression distribution of Ferroptosis-related mRNA in PsA and CL group (A), in PsA and Ps group (B). The horizontal axis represents different mRNA, the vertical axis represents the mRNA expression distribution, and the upper left corner represents the significance $p$-value test method. C and D: Correlation between hub genes and ferroptosis-related genes in PsA group (C) and Ps group (D). The horizontal axis represents top 7 hub genes, the vertical axis represents ferroptosisrelated genes. Red represents positive correlation, blue represents negative correlation, and the darker the color represents the two stronger correlation. Asterisks represent levels of significance ${ }^{\star} \mathrm{p}<0.05,{ }^{*} \mathrm{p}<$ $0.01)$.

\section{Figure 8}

Ferroptosis related analysis validation. A: Box plot after data standardization of Ps group. B: Correlation between hub genes and ferroptosis-related genes in PsA group 